\title{
The Beta Exponentiated Pareto Distribution with Application to Bladder Cancer Susceptibility
}

\author{
Luz M. Zea ${ }^{1}$, Rodrigo B. Silva ${ }^{1}$, Marcelo Bourguignon ${ }^{1}$, Andrea M. Santos ${ }^{1} \&$ Gauss M. Cordeiro ${ }^{1}$ \\ ${ }^{1}$ Departamento de Estatística, Universidade Federal de Pernambuco, Cidade Universitária, Recife, PE, Brazil \\ Correspondence: Luz M. Zea, Departamento de Estatística, Universidade Federal de Pernambuco, Cidade Univer- \\ sitária, Recife 50740-540, PE, Brazil. E-mail: milezea@gmail.com
}

Received: April 13, 2012 Accepted: April 26, 2012 Online Published: June 15, 2012

doi:10.5539/ijsp.v1n2p8 URL: http://dx.doi.org/10.5539/ijsp.v1n2p8

The research is financed by $\mathrm{CNPq}$ (Brazil) and CAPES (Brazil)

\begin{abstract}
A general class of univariate distributions generated by beta random variables, proposed by Eugene et al. (2002) and Jones (2009), has been discussed for many authors. In this paper, the beta exponentiated Pareto distribution is introduced and studied. Its density and failure rate functions can have different shapes. It contains as special models several important distributions discussed in the literature, such as the beta-Pareto and exponentiated Pareto distributions. We provide a comprehensive mathematical treatment of the distribution and derive expressions for the moments, generating and quantile functions and incomplete and L-moments. An explicit expression for Rényi entropy is obtained. The method of maximum likelihood is used for estimating the model parameters and the observed information matrix is derived. The flexibility of the new model is illustrated with an application to a real data set.
\end{abstract}

Keywords: Beta exponentiated Pareto distribution, hazard function, maximum likelihood

\section{Introduction}

The Pareto distribution was originally proposed to model the unequal distribution of wealth since he observed the way that a larger portion of the wealth of any society is owned by a smaller percentage of the people. Ever since, it plays an important role in analysing a wide range of real-world situations, not only in the field of economics. Examples of approximately Pareto distributed phenomena may be found in sizes of sand particles and clusters of Bose-Einstein condensate close to absolute zero.

There are several forms and extensions of the Pareto distribution in the literature. Pickands (1975) was the first to propose an extension of the Pareto distribution with the generalized Pareto (GP) distribution when analysing the upper tail of a distribution function. The GP has been used for modeling extreme value data because of its long tail feature (see Choulakian \& Stephens, 2001). Naturally, the Pareto distribution is a special case of the GP. The exponentiated Pareto (EP) distribution was introduced by Gupta et al. (1998) in the same settings that the generalized exponential (GE) distribution extends the exponential distribution (see Gupta \& Kundu, 1999). The EP distribution can be defined by raising the cumulative distribution function (cdf) of a Pareto distribution to a positive power. Thus, the random variavel $T$ with EP distribution has cdf given by

$$
G(t)=\left[1-\left(\frac{\beta}{t}\right)^{k}\right]^{\alpha},
$$

where $\alpha>0, \beta>0, k>0$ and, $t \geq \beta$. The corresponding probability density function (pdf) is given by

$$
g(t)=\frac{\alpha k \beta^{k}}{t^{k+1}}\left[1-\left(\frac{\beta}{t}\right)^{k}\right]^{\alpha-1} .
$$

This kind of extension has been receiving considerable attention over the last decade. See, for instance, the exponentiated Fréchet, exponentiated Weibull, exponentiated gamma and exponentiated Gumbel distributions, which extend the Fréchet, Weibull, gamma and Gumbel distributions in the same way that the GE distribution extends 
the exponential distribution. All these generalizations were proposed by Nadarajah and Kotz (2006a). In a recent paper, Silva et al. (2010) introduced the generalized exponential-geometric (GEG) distribution by raising the cumulative distribution function (cdf) of a exponenatial geometric distribuiton to a positive power, as well as the mentioned distributions before.

Akinsete et al. (2008) and Mahmoudi (2011) extended the Pareto and GP distributions by defining the beta Pareto (BP) and beta generalized Pareto (BGP) distributions, respectively, based on the class of generalized (so-called "beta-G") distributions introduced by Eugene et al. (2002). These extensions are obtained by taking any parent $\mathrm{G}$ distribution in the cdf of a beta distribution with two additional shape parameters, introducing skewness and varying tail weight. Following the same idea, many beta-type distributions were introduced and studied, see, for example, Barreto-Souza et al. (2010), Silva et al. (2010) and Cordeiro et al. (2011).

In this paper, we propose the beta exponentiated Pareto (BEP) which extends the Pareto, BP and EP distributions in the same set-up carried out by Eugene et al. (2002) with the hope that it will attract wider application in economics, additionally as in several areas of study. The univariate class of distributions, generated by a parent G, with parameters $a>0$ and $b>0$ is defined as follows

$$
F(x)=I_{G(x)}(a, b)
$$

where $I_{y}(a, b)=B_{y}(a, b) / B(a, b)$ denotes the incomplete beta function ratio and $B_{y}(a, b)=\int_{0}^{y} w^{a-1}(1-w)^{b-1} d w$ is the incomplete beta function.

In the same sense, several extensions were introduced and studied over the last years, notoriously after the works of Eugene et al. (2002) and Jones (2009). Eugene et al. (2002) introduced the beta normal distribution by taking $\mathrm{G}$ in (2) to be the normal cumulative distribution with parameters $\mu$ and $\sigma$, and then they calculated some of its first moments. Gupta and Nadarajah (2004) provided more general results for these moments. Nadarajah and Kotz (2004), Nadarajah and Gupta (2004) and Nadarajah and Kotz (2006) proposed the beta Fréchet (BF), beta Gumbel $(\mathrm{BGu})$ and beta exponential (BE) distributions by taking $\mathrm{G}(\mathrm{t})$ in Equation (2) to be the cdf of the Fréchet, Gumbel and exponential distributions, respectively. Another instance of the generalization given by (2) is the beta logistic distribution, which has been around for over 20 years (Brown et al., 2002), although it did not originate directly from this equation. Recently, Barreto-Souza et al. (2010) proposed the beta generalized exponential distribution by taking $G(t)$ in (2) to be the cdf of the exponentiated exponential (EE) distribution and discussed maximum likelihood estimation of its parameters.

The increasing usage of the EP distribution is the main motivation to introduce the BEP distribution, in addition to the fact that the current generalization provides means of its continuous extension to still more complex analysis. We derive some structural properties of the new distribution, deal with maximum likelihood estimation of the parameters and derive the observed information matrix.

The article is outlined as follows. In Section 2, we define the cumulative, density and hazard functions of the BEP distribution and list some special cases. In Section 3, we write the BEP density function as an infinite weighted sum of Pareto densities. In addition, we study the limit behaviour of its pdf and hazard rate function. A range of its mathematical properties is considered in Sections 3-6. These include generating function, incomplete moments, L-moments, quantile function and mean deviations. The Rényi entropy is determined in Section 7. In Section 8, we derive the order statistics. Maximum likelihood estimation is performed and the observed information matrix is calculated in Section 9. In Section 10, we provide an application of the BEP distribution to remission times of bladder cancer. Finally, some conclusions are addressed in Section 11.

\section{The Beta Exponentiated Pareto Distribution}

If $G(x)$ is the cdf of the EP distribution with parameters $\alpha>0, \beta>0$ and $k>0$, then Equation (2) gives the cdf of the proposed BEP distribution as

$$
F(x)=I_{\left[1-(\beta / x)^{k}\right]^{\alpha}}(a, b),
$$

for $x \geq \beta$ and $a, b>0$. The corresponding density and hazard rate functions are expressed as

$$
f(x)=\frac{\alpha k \beta^{k}}{B(a, b)} \frac{1}{x^{k+1}}\left[1-\left(\frac{\beta}{x}\right)^{k}\right]^{\alpha a-1}\left\{1-\left[1-\left(\frac{\beta}{x}\right)^{k}\right]^{\alpha}\right\}^{b-1}
$$


and

$$
\tau(x)=\frac{\alpha k \beta^{k}\left[1-(\beta / x)^{k}\right]^{\alpha a-1}\left\{1-\left[1-(\beta / x)^{k}\right]^{\alpha}\right\}^{b-1}}{x^{k+1}\left\{B(a, b)-B\left(\left[1-(\beta / x)^{k}\right]^{\alpha} ; a, b\right)\right\}},
$$

respectively. If $X$ has $\mathrm{BEP}$ distribution with parameters $a, b, \alpha, \beta$ and $k$, we denote $X \sim \operatorname{BEP}(a, b, \alpha, \beta, k)$. In Figure 1 , we plot the density and hazard rate functions for different parameters values.
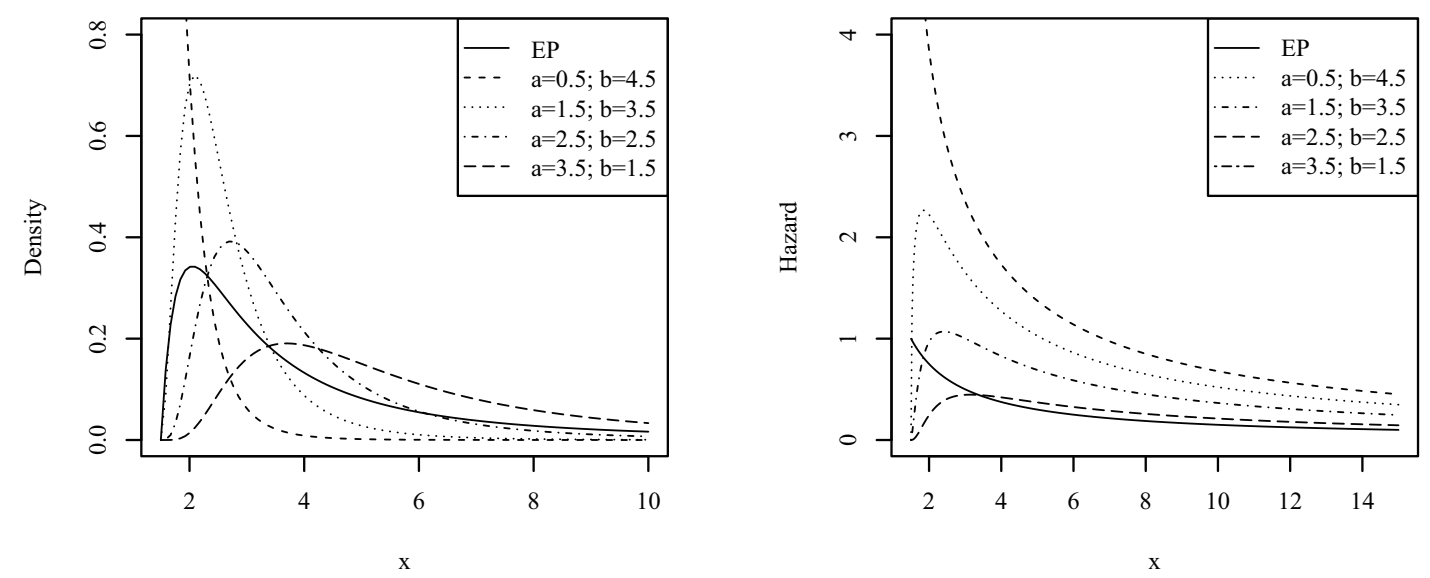

Figure 1. Plots of the BEP densities and hazard rate functions for $\alpha=2, k=0.5$ and $\beta=1.5$ and some parameter values

Note that the BEP distribution has several well known models as special cases, which make it of distinguishable scientific importance from other distributions.

- For $\alpha=1$, Equation (4) reduces to the BP distribution studied by Akinsete et al. (2008).

- By setting $a=b=\alpha=1$ or $a=1 / \alpha$ and $b=1$, we obtain the Pareto distribution with parameters $\beta$ and $k$. Furthermore, if $\alpha=a=1$, the BEP distribution becomes the Pareto distribution with parameters $\beta$ and $k b$.

- By setting $a=b=1$, Equation (4) reduces to the Pareto distribution in (1). Furthermore, if we take $b=1$, then Equation (4) reduces to Equation (1) with parameters $\alpha a, \beta$ and $k$. This case shows non-identifiability.

- If $X \sim \operatorname{BEP}(a, b, \alpha, \beta, k)$, then the random variable $Y=1-(\beta / X)^{k}$ has the Weibullized beta distribution (see Johnson et al., 1994, p. 248).

Proof: Using the transformation method, the random variable $Y$ has density function given by

$$
g(y)=\frac{\alpha}{B(a, b)} y^{\alpha(a-1)}\left(1-y^{\alpha}\right)^{b-1}, \quad 0<y<1 .
$$

- If $X \sim \operatorname{BEP}(a=1 / 2, b=1 / 2, \alpha=1, \beta, k)$, then the random variable $Y=(X / \beta)^{-k}$ has the arcsine distribution.

- If $X \sim \operatorname{BEP}(a, b, \alpha=1, \beta, k)$, then the random variable $Y=b \log (X / \beta)$ has the $\log$-beta distribution with parameters $a, k$ and $k / b$.

- If $X \sim \operatorname{BEP}(a=1, b, \alpha=1, \beta, k)$, then the random variable $Y=b \log (X / \beta)$ follows the exponential distribution with mean $1 / k$.

Proof: The proofs of the last three items can be obtained from Akinsete et al. (2008).

- If $X \sim \operatorname{BEP}(a, b, \alpha=1, \beta, k)$, then the pdf of the random variable $Y=b \log (X / \beta)$ is an integral representation of a beta function when $k=b$, or a special case of the beta-Weibull distribution, $\mathrm{BW}(a, b, c, \gamma)$.

\section{Properties of the BEP Distribution}

In this section, we analyse some properties of the BEP distribution such as the asymptotic behavior of density and hazard functions. Furthermore, we provide the BEP density as a infinite weighted sum of Pareto densities. 
From Equation (4), it is easily seen that the limit of the BEP density function as $x \rightarrow \infty$ is 0 and

$$
f(x)= \begin{cases}\infty, & \text { for } 0<\alpha<1 / a \\ \frac{\alpha k b}{\beta}, & \text { for } \alpha=1 / a \\ 0, & \text { for } \alpha>1 / a\end{cases}
$$

as $x$ approaches $\beta$.

In a similar way, the BEP hazard function approaches zero when $x \rightarrow \infty$ and

$$
\tau(x)= \begin{cases}\infty, & \text { for } 0<\alpha<1 / a \\ \frac{\alpha k b}{\beta}, & \text { for } \alpha=1 / a \\ 0, & \text { for } \alpha>1 / a .\end{cases}
$$

as $x$ approaches $\beta$. This result is straightforward from Equation (5).

We now give an interesting and useful expansion for the BEP density (4). For any positive real number $r$, and for $|z|<1$, a generalized binomial expansion holds

$$
(1-z)^{-r}=\sum_{j=0}^{\infty} \frac{\Gamma(r+j)}{\Gamma(r) j !} z^{j}
$$

By using this in the last term of Equation (4), we can write

$$
f(x)=\frac{\alpha k \beta^{k}}{B(a, b)} \frac{1}{x^{k+1}} \sum_{i=0}^{\infty}(-1)^{i}\left(\begin{array}{c}
b-1 \\
i
\end{array}\right)\left[1-\left(\frac{\beta}{x}\right)^{k}\right]^{\alpha(i+a)-1} .
$$

Again, by using (6) in the last factor of each summand in the last equation, we obtain

$$
f(x)=\frac{\alpha k}{B(a, b)} \sum_{j=0}^{\infty} \sum_{i=0}^{\infty}(-1)^{i+j}\left(\begin{array}{c}
b-1 \\
i
\end{array}\right)\left(\begin{array}{c}
\alpha(i+a)-1 \\
j
\end{array}\right) \frac{\beta^{k(j+i)}}{x^{k(j+1)+1}},
$$

and then

$$
f(x)=\sum_{j=0}^{\infty} w_{j} h(x ; \beta, k(j+1))
$$

where

$$
w_{j}=\frac{\alpha}{B(a, b)} \sum_{i=0}^{\infty} \frac{(-1)^{i+j}}{j+1}\left(\begin{array}{c}
b-1 \\
i
\end{array}\right)\left(\begin{array}{c}
\alpha(i+a)-1 \\
j
\end{array}\right)
$$

and $h(x ; \beta, k(j+1))$ denotes the Pareto density with parameters $\beta$ and $k(j+1)$. Equation (7) is the main result of this section.

The above result ensures that some mathematical properties such as ordinary and incomplete moments, generating function and mean deviations can be derived from those quantities of the reparametrized (with parameters $\beta$ and $k(j+1))$ Pareto distribution.

\section{Moments}

Here after, let $X$ be a random variable having the BEP distribution (3). Using Equation (7), it is easy to obtain the $r$ th moment of $X$. If we assume that $Y$ is a Pareto distributed random variable, then the $r$ th moment of $Y$ is given by

$$
\mathrm{E}\left(Y^{r}\right)=\frac{k \beta^{r}}{k-r}, \quad r<k
$$

From Equation (7), we obtain

$$
\mathrm{E}\left(X^{r}\right)=k \beta^{r} \sum_{j=0}^{\infty} \frac{(j+1) w_{j}}{[k(j+1)-r]},
$$


whenever $r<k$. By setting $r=1$ in Equation (8), gives the mean of $X$

$$
\mu=\mathrm{E}(X)=\sum_{j=0}^{\infty} w_{j} \frac{k(j+1) \beta}{[k(j+1)-1]},
$$

whenever $k>1$. Setting $\alpha=a=b=1$, we obtain

$$
w_{j}= \begin{cases}1, & \text { for } j=0 \\ 0, & \text { for } j \geq 1\end{cases}
$$

So, the mean $\mu$ reduces to

$$
\mathrm{E}(X)=\frac{k \beta}{k-1},
$$

which is precisely the mean of the Pareto distribution.

\subsection{Moment Generating Function}

The moment generating function (mgf) $M_{Y}(t)$ corresponding to a random variable $Y$ with Pareto distribution with parameters $\beta$ and $k$ is only defined for negative values of $t$. It is given by

$$
M_{Y}(t)=k(-\beta t)^{k} \Gamma(-k,-\beta t)
$$

where $\Gamma(\cdot, \cdot)$ denotes the incomplete gamma function:

$$
\Gamma(s, x)=\int_{x}^{\infty} t^{s-1} \mathrm{e}^{-t} d t
$$

From Equation (7), the mgf $M_{X}(t)$ of $X$ reduces to

$$
M_{X}(t)=k(-\beta t)^{k} \sum_{j=0}^{\infty} w_{j}(j+1)(-\beta t)^{k j} \Gamma(-k(j+1),-\beta t), \text { for } t<0 .
$$

\subsection{Incomplete Moments}

If $Y$ is a random variable with Pareto distribution with parameters $\beta$ and $k$, the $r$ th incomplete moment, for $r<k$, is given by

$$
m_{r}(y)=\frac{k \beta^{r}}{k-r}\left[1-\left(\frac{\beta}{y}\right)^{k-r}\right]
$$

From this formula we verify that $m_{r}(y) \rightarrow E\left(Y^{r}\right)$ when $y \rightarrow \infty$, whenever $r<k$. So, the $r$ th incomplete moment of $X$ follows from Equation (7) as

$$
m_{r}(y)=\sum_{j=0}^{\infty} w_{j} \int_{\beta}^{y} x^{r} h(x ; \beta, k(j+1)) d x=k \beta^{r} \sum_{j=0}^{\infty} \frac{(j+1) w_{j}}{[k(j+1)-r]}\left[1-\left(\frac{\beta}{y}\right)^{k(j+1)-r}\right],
$$

where the last equality holds provided that $r<k$.

\subsection{L-moments}

The L-moments are equivalent to the ordinary moments but can be estimated by linear combinations of order statistics. These quantities are linear functions of expected order statistics defined by

$$
\lambda_{m+1}=\frac{m}{(m+1)} \sum_{k=0}^{m} \frac{(-1)^{k}}{k} \mathrm{E}\left(X_{m+1-k: m+1}\right), r=0,1, \ldots
$$

The first four L-moments are: $\lambda_{1}=\mathrm{E}\left(X_{1: 1}\right), \lambda_{2}=\frac{1}{2} \mathrm{E}\left(X_{2: 2}-X_{1: 2}\right), \lambda_{3}=\frac{1}{3} \mathrm{E}\left(X_{3: 3}-2 X_{2: 3}+X_{1: 3}\right)$ and $\lambda_{4}=$ $\frac{1}{4} \mathrm{E}\left(X_{4: 4}-3 X_{3: 4}+3 X_{2: 4}-X_{1: 4}\right)$. The L-moments have the asset that they exist whenever the mean of the distribution exists, in despite of some higher moments may not exist, and are relatively robust to the effects of outliers. 
From Equation (11) the expansion for the means of the order statistics corresponding to $r=1$, we can easily obtain the L-moments of the BEP distribution.

\section{Quantile Function}

The quantile function corresponding to (3) is

$$
Q(u)=F^{-1}(u)=\frac{\beta}{\left\{1-\left[I_{u}^{-1}(a, b)\right]^{1 / \alpha}\right\}^{1 / k}}, 0<u<1,
$$

where $I_{u}^{-1}(a, b)$ is the inverse of the incomplete beta function. The function $I_{u}^{-1}(a, b)$ can be expressed as a power series

$$
I_{u}^{-1}(a, b)=\sum_{i=1}^{\infty} q_{i}[a B(a, b) u]^{i / a},
$$

where $q_{1}=1$ and the remaining coefficients satisfy the following recursion

$$
\begin{aligned}
q_{i}= & \frac{1}{i^{2}+(a-2) i+(1-a)}\left\{\left(1-\delta_{i, 2}\right) \sum_{r=2}^{i-1} q_{r} q_{i+1-r}[r(1-a)(i-r)-r(r-1)]+\right. \\
& \left.\sum_{r=1}^{i-1} \sum_{s=1}^{i-r} q_{r} q_{s} q_{i+1-r-s}[r(r-a)+s(a+b-2)(i+1-r-s)]\right\},
\end{aligned}
$$

where $\delta_{i, 2}=1$ if $i=2$ and $\delta_{i, 2}=0$ if $i \neq 2$. The shortcomings of the classical kurtosis measure are well-known. There are many heavy-tailed distributions for which this quantity is infinite. So, it becomes uninformative precisely when it needs to be. Indeed, our motivation to use quantile-based measures stemmed from the non-existence of classical kurtosis for many generalized distributions.

The Bowley's skewness is based on quartiles, Kenney and Keeping (1962):

$$
B=\frac{Q(3 / 4)-2 Q(1 / 2)+Q(1 / 4)}{Q(3 / 4)-Q(1 / 4)}
$$

and the Moors' kurtosis (Moors, 1998) is based on octiles:

$$
M=\frac{Q(7 / 8)-Q(5 / 8)-Q(3 / 8)+Q(1 / 8)}{Q(6 / 8)-Q(2 / 8)},
$$

where $Q(\cdot)$ represents the quantile function define in (9). Plots of the skewness and kurtosis for some choices of the parameter $b$ as functions of $a$, and for some choices of $a$ as functions of $b$, for $\alpha=1, \beta=0.5$ and $k=0.5$, are shown in Figure 2. These plots indicate that the skewness and kurtosis decrease when $b$ increases for fixed $a$ and when $a$ increases for fixed $b$. 

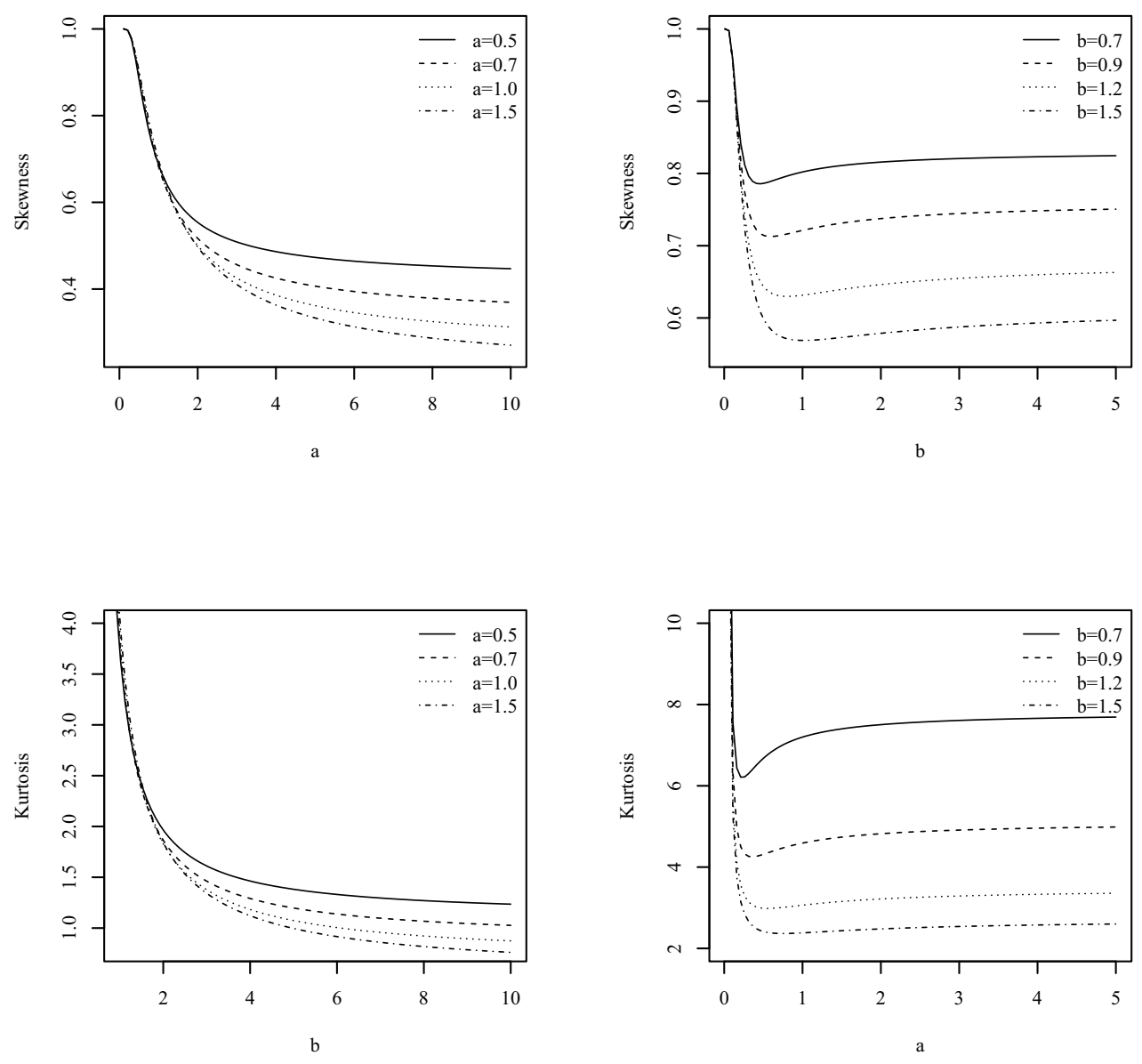

Figure 2. Plots of the BEP skewness and kurtosis as functions of a for selected values of $b$ and as functions of $b$ for selected values of a

\section{Mean Deviations}

The deviation from the mean and deviation from the median are usually used as a measure of spread in a population. Let $\mu=\mathrm{E}(X)$ and $\theta$ be the mean and the median of the BEP distribution, respectively. The mean deviations about the mean and about the median of $X$ can be calculated as

$$
D(\mu)=\int_{\beta}^{\infty}|x-\mu| f(x) d x=2 \mu F(\mu)-2 m_{1}(\mu)
$$

and

$$
D(\theta)=\int_{\beta}^{\infty}|x-\theta| f(x) d x=\mu-2 m_{1}(\theta),
$$

respectively, where $F(\mu)$ follows from (3) and $m_{1}(\mu)$ denotes the first incomplete moment.

\section{Rényi Entropy}

In the present section, we provide the Rényi entropy, which is a measure of variation of the uncertainty. The theory of entropy has been successfully used in a wide diversity of applications and has also been used for the characterization of numerous standard probability distributions. For the density function $f(x)$, the Rényi entropy is defined by

$$
I_{R}(\delta)=\frac{1}{1-\delta} \log \{I(\delta)\},
$$


where $I(\delta)=\int f^{\delta}(x) d x, \delta>0$ and $\delta \neq 1$. Using the BEP density, we obtain

$$
I(\delta)=\frac{\alpha^{\delta} k^{\delta} \beta^{k \delta}}{B^{\delta}(a, b)} \int_{\beta}^{\infty} \frac{1}{x^{(k+1) \delta}}\left[1-(\beta / x)^{k}\right]^{\delta(\alpha a-1)}\left\{1-\left[1-(\beta / x)^{k}\right]^{\alpha}\right\}^{\delta(b-1)} d x .
$$

Applying the binomial expansion to the last factor in the above integrand yields

$$
I(\delta)=\frac{\alpha^{\delta} k^{\delta} \beta^{k \delta}}{B^{\delta}(a, b)} \int_{\beta}^{\infty} \frac{1}{x^{(k+1) \delta}}\left[1-(\beta / x)^{k}\right]^{\delta(\alpha a-1)} \sum_{j=0}^{\infty}(-1)^{j}\left(\begin{array}{c}
\delta(b-1) \\
j
\end{array}\right)\left[1-(\beta / x)^{k}\right]^{\alpha j} d x
$$

Changing variables and simplifying, $I(\delta)$ reduces to

$$
I(\delta)=\frac{\alpha^{\delta} k^{\delta-1}}{\beta^{\delta-1} B^{\delta}(a, b)} \sum_{j=0}^{\infty}(-1)^{j}\left(\begin{array}{c}
\delta(b-1) \\
j
\end{array}\right) B\left[(\delta-1)\left(\frac{k+1}{k}\right)+1, \delta(a \alpha-1)+\alpha j+1\right] .
$$

Hence, the formula for the Rényi entropy becomes

$$
I_{R}(\delta)=\log \left(\frac{\beta}{k}\right)+(1-\delta)^{-1}\left\{\delta \log \left(\frac{\alpha}{B(a, b)}\right)+\log \left[\sum_{j=0}^{\infty}(-1)^{j}\left(\begin{array}{c}
\delta(b-1) \\
j
\end{array}\right) B\left(\frac{(\delta-1)(k+1)}{k}+1, \delta(a \alpha-1)+j \alpha+1\right)\right]\right\} .
$$

\section{Order Statistics}

This Section shall be concerned with order statistics, which play an important supporting role in several applications such as the statistical study of floods and droughts, in problems of breaking strength and fatigue failure. We now derive an explicit expression for the density function of the $i$ th order statistic $X_{i: n}$, say $f_{i: n}(x)$, in a random sample of size $n$ from the BEP distribution. We can write

$$
f_{i: n}(x)=\frac{f(x)}{B(i, n-i+1)} \sum_{l=0}^{n-i}(-1)^{l}\left(\begin{array}{c}
n-i \\
l
\end{array}\right) F^{i+l-1}(x) .
$$

For $b$ real non-integer, we have the series representation for the incomplete beta function

$$
B_{x}(a, b)=x^{a} \sum_{m=0}^{\infty} \frac{(1-b)_{m} x^{m}}{(a+m) m !} .
$$

Using (6) and (10), we can express (3) as $F(x)=\sum_{r=0}^{\infty} c_{r} z^{r}$, where

$$
c_{r}=\sum_{m=0}^{\infty}(-1)^{r} \delta_{m}\left(\begin{array}{c}
\alpha(a+m) \\
r
\end{array}\right)
$$

where $\delta_{m}=(1-b)_{m} /[B(a, b)(a+m) m !]$ and $z=\left(\frac{\beta}{x}\right)^{k}$. Using the identity $\left(\sum_{r=0}^{\infty} c_{r} z^{r}\right)^{n}=\sum_{r=0}^{\infty} d_{r} z^{r}$ (see Gradshteyn \& Ryzhik, 2000), where $d_{0}=c_{0}^{i+l-1}$ and

$$
d_{s}=\left(s c_{0}\right)^{-1} \sum_{r=1}^{s}[r(i+l)-s] c_{r} d_{s-k}
$$

for $s=1,2, \ldots$, the pdf of the $i$ th order statistic can be written as

$$
f_{i: n}(x)=\sum_{j, r=0}^{\infty} w_{j, r}^{*} h(x ; \beta, k(j+r+1))
$$

where

$$
w_{j, r}^{*}=\sum_{l=0}^{n-i} \frac{(-1)^{l}(j+1) w_{j} d_{r}\left(\begin{array}{c}
n-i \\
l
\end{array}\right)}{(j+r+1) B(i, n-i+1)}
$$


and $h(x ; \beta, k(j+r+1))$ denotes a Pareto density function with parameters $\beta$ and $k(j+r+1)$. Hence, some mathematical properties of the Pareto order statistics can be immediately obtained from Equation (11) and those properties of the Pareto distribution, including the sth moment $\mathrm{E}\left(X_{i: n}^{s}\right)$.

\section{Estimation and Fisher Information Matrix}

In this section, we examine estimation by maximum likelihood and inference for the BEP distribution. Let $X_{1}, \ldots, X_{n}$ be a random sample from $X \sim \operatorname{BEP}(a, b, \alpha, \beta, k)$ with observed values $x_{1}, \ldots, x_{n}$ and let $\boldsymbol{\theta}=(a, b, \alpha, \beta, k)^{T}$ be the vector of the model parameters. The $\log$-likelihood function for $\boldsymbol{\theta}$ reduces to

$$
\begin{aligned}
\ell(\boldsymbol{\theta}) & =n \log (\alpha)+n \log (k)+n k \log (\beta)-n \log [B(a, b)]-(k+1) \sum_{i=1}^{n} \log \left(x_{i}\right) \\
& +(\alpha a-1) \sum_{i=1}^{n} \log \left[1-\left(\frac{\beta}{x_{i}}\right)^{k}\right]+(b-1) \sum_{i=1}^{n} \log \left\{1-\left[1-\left(\frac{\beta}{x_{i}}\right)^{k}\right]^{\alpha}\right\}
\end{aligned}
$$

The score vector is $U(\boldsymbol{\theta})=(\partial \ell / \partial \alpha, \partial \ell / \partial k, \partial \ell / \partial a, \partial \ell / \partial b)^{T}$, where the score components corresponding to the model parameters are calculated by differentiating (12). By setting $z_{i}=1-\left(\beta / x_{i}\right)^{k}$, we obtain

$$
\begin{aligned}
\frac{\partial \ell}{\partial \alpha} & =\frac{n}{\alpha}+a \sum_{i=1}^{n} \log \left(z_{i}\right)+(b-1) \sum_{i=1}^{n} \frac{z_{i}^{\alpha} \log \left(z_{i}\right)}{1-z_{i}^{\alpha}} \\
\frac{\partial \ell}{\partial k} & =\frac{n}{k}+\frac{1}{k} \sum_{i=1}^{n} \log \left(1-z_{i}\right)-\frac{(\alpha a-1)}{k} \sum_{i=1}^{n} \frac{\left(1-z_{i}\right) \log \left(1-z_{i}\right)}{z_{i}} \\
& +\frac{\alpha(b-1)}{k} \sum_{i=1}^{n} \frac{\left(1-z_{i}\right) \log \left(1-z_{i}\right) z_{i}^{\alpha-1}}{1-z_{i}^{\alpha}} \\
\frac{\partial \ell}{\partial a} & =n[\psi(a+b)-\psi(a)]+\alpha \sum_{i=1}^{n} \log \left(z_{i}\right) \\
\frac{\partial \ell}{\partial b} & =n[\psi(a+b)-\psi(b)]+\sum_{i=1}^{n} \log \left(1-z_{i}^{\alpha}\right)
\end{aligned}
$$

where $\psi(\cdot)$ is the digamma function.

The maximum likelihood estimates (MLEs) of the parameters can be obtained by solving iteratively Equations (13)-(16). Since $x \geq \beta$, the MLE of $\beta$ is the first-order statistic $x_{(1)}$. For interval estimation and hypothesis tests on the model parameters, we require the observed information matrix

$$
K_{n}(\theta)=n\left[\begin{array}{cccc}
\kappa_{\alpha \alpha} & \kappa_{\alpha k} & \kappa_{\alpha a} & \kappa_{\alpha b} \\
\kappa_{k \alpha} & \kappa_{k k} & \kappa_{k a} & \kappa_{k b} \\
\kappa_{a \alpha} & \kappa_{a k} & \kappa_{a a} & \kappa_{a b} \\
\kappa_{b \alpha} & \kappa_{b k} & \kappa_{b a} & \kappa_{b b}
\end{array}\right]
$$

whose entries are obtained from standard calculations:

$$
\begin{aligned}
& \kappa_{\alpha \alpha}=-\frac{n}{\alpha^{2}}-(b-1) \sum_{i=1}^{n} \frac{\log ^{2}\left(z_{i}\right) z_{i}^{\alpha}}{\left(1-z_{i}^{\alpha}\right)^{2}}, \quad \kappa_{\alpha a}=\sum_{i=1}^{n} \log \left(z_{i}\right), \quad \kappa_{\alpha b}=-\sum_{i=1}^{n} \frac{z_{i}^{\alpha} \log \left(z_{i}\right)}{1-z_{i}^{\alpha}}, \\
& \kappa_{\alpha k}=\frac{(b-1)}{k} \sum_{i=1}^{n} \frac{\left(1-z_{i}\right) \log \left(1-z_{i}\right) z_{i}^{\alpha-1\left(1-z_{i}^{\alpha}+\log z_{i}^{\alpha}\right)}}{\left(1-z_{i}^{\alpha}\right)^{2}}-\frac{a}{k} \sum_{i=1}^{n} \frac{\left(1-z_{i}\right) \log \left(1-z_{i}\right)}{z_{i}} \\
& \kappa_{k k}=-\frac{n}{k^{2}}-\frac{(\alpha a-1)}{k^{2}} \sum_{i=1}^{n} \frac{\left(1-z_{i}\right) \log ^{2}\left(1-z_{i}\right)}{z_{i}^{2}} \\
& +\frac{\alpha(b-1)}{k^{2}} \sum_{i=1}^{n} \frac{\left[1-\alpha\left(1-z_{i}\right)-z_{i}^{\alpha}\right] z_{i}^{\alpha-2}\left(1-z_{i}\right) \log ^{2}\left(1-z_{i}\right)}{\left(1-z_{i}^{\alpha}\right)^{2}}
\end{aligned}
$$




$$
\begin{aligned}
& \kappa_{a a}=n\left[\psi^{\prime}(a+b)-\psi^{\prime}(a)\right], \quad \kappa_{b b}=n\left[\psi^{\prime}(a+b)-\psi^{\prime}(b)\right], \\
& \kappa_{a k}=-\frac{\alpha}{k} \sum_{i=1}^{n} \frac{\left(1-z_{i}\right) \log \left(1-z_{i}\right)}{z_{i}}, \quad \kappa_{a b}=n \psi^{\prime}(a+b), \quad \kappa_{b k}=\frac{\alpha}{k} \sum_{i=1}^{n} \frac{z_{i}^{\alpha-1}\left(1-z_{i}\right) \log \left(1-z_{i}\right)}{1-z_{i}^{\alpha}} .
\end{aligned}
$$

\section{Application}

Here, we use a real data set to compare the fits of the BEP distribution and those of other sub-models, i.e., the beta Pareto (BP), EP and Pareto distributions. We make a results comparison of the models fit. We consider an uncensored data set corresponding to remission times (in months) of a random sample of 128 bladder cancer patients. These data were previously studied by Lee and Wang (2003) and Lemonte and Cordeiro (2011). Bladder cancer is a disease in which abnormal cells multiply without control in the bladder. The most common type of bladder cancer recapitulates the normal histology of the urothelium and is known as transitional cell carcinoma.The data are as follows: $0.08,0.20,0.40,0.50,0.51,0.81,0.90,1.05,1.19,1.26,1.35,1.40,1.46,1.76,2.02,2.02$, $2.07,2.09,2.23,2.26,2.46,2.54,2.62,2.64,2.69,2.69,2.75,2.83,2.87,3.02,3.25,3.31,3.36,3.36,3.48,3.52$, $3.57,3.64,3.70,3.82,3.88,4.18,4.23,4.26,4.33,4.34,4.40,4.50,4.51,4.87,4.98,5.06,5.09,5.17,5.32,5.32$, $5.34,5.41,5.41,5.49,5.62,5.71,5.85,6.25,6.54,6.76,6.93,6.94,6.97,7.09,7.26,7.28,7.32,7.39,7.59,7.62$, $7.63,7.66,7.87,7.93,8.26,8.37,8.53,8.65,8.66,9.02,9.22,9.47,9.74,10.06,10.34,10.66,10.75,11.25$, $11.64,11.79,11.98,12.02,12.03,12.07,12.63,13.11,13.29,13.80,14.24,14.76,14.77,14.83,15.96,16.62$, $17.12,17.14,17.36,18.10,19.13,20.28,21.73,22.69,23.63,25.74,25.82,26.31,32.15,34.26,36.66,43.01$, $46.12,79.05$. These remission times are a subset of the bladder cancer data and are used here only for illustrative purposes. The required numerical evaluations are carried out using the SAS (PROCNLMIXED) and R softwares.

Tables 1 and 2 provide some descriptive statistics and the MLEs (with corresponding standard errors in parentheses) of the model parameters. The model selection is carried out using the AIC (Akaike information criterion), the BIC (Bayesian information criterion) and the CAIC (consistent Akaike information criteria):

$$
\mathrm{AIC}=-2 \ell(\hat{\boldsymbol{\theta}})+2 q, \quad \mathrm{BIC}=-2 \ell(\hat{\boldsymbol{\theta}})+q \log (n) \text { and } \mathrm{CAIC}=-2 \ell(\hat{\boldsymbol{\theta}})+\frac{2 q n}{n-q-1},
$$

where $\ell(\hat{\boldsymbol{\theta}})$ denotes the log-likelihood function evaluated at the maximum likelihood estimates, $q$ is the number of parameters, and $n$ is the sample size.

Table 1. Descriptive statistics for the remission times of bladder cancer data

\begin{tabular}{ccccccccc}
\hline Min. & $Q_{1}$ & $Q_{2}$ & Mean & $Q_{3}$ & Max. & Var. & Skewness & Kurtosis \\
0.080 & 3.348 & 6.395 & 9.366 & 11.840 & 79.050 & 110.425 & 3.248 & 15.195 \\
\hline
\end{tabular}

\begin{tabular}{|c|c|c|c|c|c|c|c|c|}
\hline \multirow[b]{2}{*}{ Model } & \multicolumn{3}{|c|}{ Estimates } & & & \multicolumn{2}{|c|}{ Statistic } & \multirow[b]{2}{*}{ CAIC } \\
\hline & $\hat{a}$ & $\hat{b}$ & $\hat{k}$ & $\hat{\beta}$ & $\hat{\alpha}$ & AIC & BIC & \\
\hline \multirow[t]{2}{*}{ BEP } & 0.3477 & 159831 & 0.05080 & 0.0800 & 8.6121 & 874.8 & 886.2 & 875.1 \\
\hline & $(0.0965)$ & $(183.7501)$ & $(0.0185)$ & & $(2.0925)$ & & & \\
\hline \multirow[t]{2}{*}{$\mathrm{BP}$} & 4.8049 & 100.5023 & 0.0109 & 0.0800 & & 970.7 & 979.2 & 970.9 \\
\hline & $(0.0552)$ & $(0.2512)$ & $(0.0005)$ & & & & & \\
\hline \multirow[t]{2}{*}{$\mathrm{EP}$} & & & 0.4722 & 0.0800 & 4.1518 & 992.2 & 997.9 & 992.3 \\
\hline & & & $(0.0247)$ & & $(0.5508)$ & & & \\
\hline \multirow[t]{2}{*}{ Pareto } & & & 0.1519 & 0.0800 & & 1189.3 & 1192.1 & 1189.3 \\
\hline & & & $(0.0134)$ & & & & & \\
\hline
\end{tabular}

Table 2. MLEs of the model parameters, the corresponding SEs (given in parentheses) and the statistics AIC, BIC and CAIC

Since the values of the AIC, BIC and CAIC are smaller for the BEP distribution compared with those values of the other models, the new distribution seems to be a very competitive model to these data.

Plots of the estimated pdf and cdf of the BEP, BP, EP and Pareto models fitted to these data are given in Figure 3. They indicate that the BEP distribution is superior to the other distributions in terms of model fitting.

Table 3 lists the values of the Kolmogorov-Smirnov (K-S) statistic and of $-2 \ell(\hat{\boldsymbol{\theta}})$. From these figures, we conclude that the BEP distribution provides a better fit to these data than the BP, EP and Pareto models. 

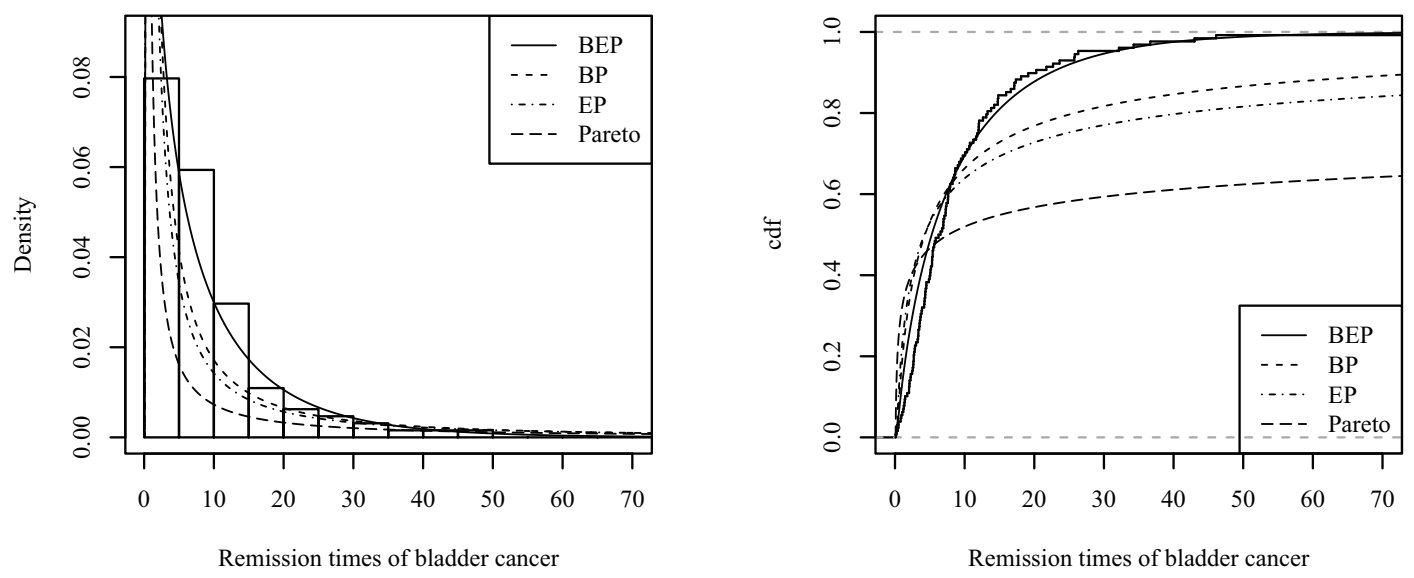

Figure 3. Estimated pdf (a) and cdf (b) of the BEP, BP, EP and Pareto models for the remission times of bladder cancer data

Table 3. K-S and $-2 \ell(\hat{\boldsymbol{\theta}})$ statistics for the remission times of bladder cancer data

\begin{tabular}{lcccc}
\hline Model & BEP & BP & EP & Pareto \\
\hline K-S & 0.1427 & 0.2231 & 0.2515 & 0.3729 \\
$-2 \ell(\widehat{\theta})$ & 866.8 & 964.7 & 988.2 & 1187.3 \\
\hline
\end{tabular}

In summary, the proposed BEP distribution produces better fits to the data than its sub-models.

\section{Concluding Remarks}

The well-known three-parameter exponentiated Pareto distribution, introduced by Gupta et al. (1998), is extended by introducing two extra shape parameters, thus defining the beta exponentiated Pareto (BEP) distribution having a broader class of hazard rate and density functions. This is achieved by taking (1) as the baseline cumulative distribution of the generalized class of beta distributions defined by Eugene et al. (2002). A detailed study on the mathematical properties of the new distribution is presented. The new model includes as special sub-models the Pareto, exponentiated Pareto (EP) (Gupta et al., 1998) and beta Pareto (BP) (Mahmoudi, 2011) distributions. We obtain the moment generating function, ordinary moments, quantile function, order statistics and Rényi entropy. The estimation of the model parameters is approached by maximum likelihood and the observed information matrix is obtained. An application to a real data set indicates that the fit of the new model is superior to the fits of its principal sub-models. We hope that the proposed model may be interesting for a wider range of statistical research.

\section{Acknowledgment}

We gratefully acknowledge the grants from CAPES and CNPq (Brazil). The authors are also grateful to an associate editor and two referees for helpful comments and suggestions.

\section{References}

Akinsete, A., Famoye, F., \& Lee, C. (2008). The beta-Pareto distribution. Statistics, 42(6), 547-563. http://dx.doi.org/10.1080/02331880801983876

Barreto-Souza, W., Santos, A. H. S., \& Cordeiro, G. M. (2010). The beta generalized exponential distribution. $\begin{array}{llrr}\text { Journal of Statistical Computation and Simulation, } & 80,\end{array}$ http://dx.doi.org/10.1080/00949650802552402

Brown, B. W., Floyd, M. S., \& Levy, L. B. (2002). The log-F: a distribution for all seasons. Computational Statistics, 17, 47-58.

Choulakian, V., \& Stephens, M. A. (2001). Goodness-of-fit for the generalized Pareto distribution. Technometrics, 
43, 478-484. http://dx.doi.org/10.1198/00401700152672573

Cordeiro, G. M., Silva, G. O., \& Ortega, E. M. M. (2011). The beta-Weibull geometric distribution. Statistics, 1-18. http://dx.doi.org/10.1080/02331888.2011.577897

Eugene, N., Lee, C., \& Famoye, F. (2002). Beta-normal distribution and its applications. Communications in Statistics: Theory and Methods, 31, 497-512. http://dx.doi.org/10.1081/STA-120003130

Gradshteyn, I. S., \& Ryzhik, I. M. (2000). Table of Integrals, Series, and Products. San Diego: Academic Press.

Gupta, R. D., \& Kundu, D. (1999). Generalized exponential distributions. Austral. NZ J. Statist., 41, $173-188$. http://dx.doi.org/10.1111/1467-842X.00072

Gupta, R. C., Gupta, R. D., \& Gupta, P. L. (1998). Modeling failure time data by Lehman alternatives. Communications in Statistics: Theory and Methods, 27, 887-904. http://dx.doi.org/10.1080/03610929808832134

Gupta, A. K., \& Nadarajah, S. (2004). On the moments of the beta normal distribution. Communications in Statistics - Theory and Methods, 33, 1-13. http://dx.doi.org/10.1081/STA-120026573

Jones, M. C. (2009). A beta-type distribution with some tractability advantages. Statistical Methodology, 6, 70-81. http://dx.doi.org/10.1016/j.stamet.2008.04.001

Kenney, J. F., \& Keeping, E. S. (1962). Mathematics of Statistics (3rd ed.), Part 1. New Jersey.

Johnson, N. L., Kotz, S., \& Balakrishnan, N. (1994). Continuous Univariate Distributions (2nd ed.), Vol. 1. New York: Wiley.

Lee, E. T., \& Wang, J. W. (2003). Statistical Methods for Survival Data Analysis (3rd ed.). New York: Wiley.

Lemonte, A. J., \& Cordeiro, G. M. (2011). An extended Lomax distribution. Statistics, 1-17. http://dx.doi.org/10.1080/02331888.2011.568119

Mahmoudi, E. (2011). The beta generalized Pareto distribution with application to lifetime data. Mathematics and Computers in Simulation, 81, 2414-2430. http://dx.doi.org/10.1016/j.matcom.2011.03.006

Moors, J. J. (1988). A quantile alternative for kurtosis. Journal of the Royal Statistical Society D, 37, $25-32$. http://dx.doi.org/10.2307/2348376

Nadarajah, S., \& Gupta, A. K. (2004). The beta Frechét distribution. Far East J. Theo. Stat., 14, 15-24.

Nadarajah, S., \& Kotz, S. (2006a). The exponentiated type distributions. Acta Appl. Math., 92, 97-111. http://dx.doi.org/10.1007/s10440-006-9055-0

Nadarajah, S., \& Kotz, S. (2004). The beta Gumbel distribution. Math. Probab. Eng., 10, 323-332. http://dx.doi.org/10.1155/S1024123X04403068

Pickands, J. (1975). Statistical inference using extreme order statistics. Annals of Statistics, 3, 119-131.

Silva, G. O., Ortega, E. M. M., \& Cordeiro, G. M. (2010). The beta modified Weibull distribution. Lifetime Data Anal., 16, 409-430. http://dx.doi.org/10.1007/s10985-010-9161-1

Silva, R. B., Barreto-Souza, W., \& Cordeiro, G. M. (2010). A new distribution with decreasing, increasing and upside-down bathtub failure rate. Comput. Statist. Data Anal., 54, 935-934. http://dx.doi.org/10.1016/j.csda.2009.10.006 